\title{
Erdheim-Chester Disease With Renal Involvement: A Case Report
}

\author{
Ali Kürşat GANIYUSUFOĞLU, ${ }^{1}$ Gökşen GÖKŞENOĞLU, ${ }^{1}$ Burçin TUNÇ, ${ }^{2}$ Nurdan PAKER ${ }^{1}$ \\ ${ }^{1}$ Department of Physical Medicine and Rehabilitation, \\ İstanbul Physical Medicine and Rehabilitation Training Research Hospital, İstanbul, Turkey \\ ${ }^{2}$ Department of Radiology, Ethica İncirli Hospital, İstanbul, Turkey
}

\begin{abstract}
Erdheim-Chester disease is a type of non-Langerhans cell histiocytosis. It is a rare, multisystem disorder with unknown etiology. Heterogeneity of the clinical symptoms makes the diagnosis challenging. On the other hand, knowing the signs and radiological findings of the disease helps to establish a correct diagnosis. In this article, we present a 51-year-old male patient with skeletal and urinary system manifestations who finally underwent right nephrectomy due to renal insufficiency. The diagnosis was suspected by the retroperitoneal infiltrative process and radiological findings of the tubular bones. Erdheim-Chester disease diagnosis was confirmed with CD68(+) CD1a(-) histiocytes detected by immunohistochemical analysis of the nephrectomy specimen.

Keywords: Bone diseases; Erdheim-chester disease; non-Langerhans cell histiocytosis.
\end{abstract}

Erdheim-Chester disease (ECD) is one of the nonLangerhans cell histiocytosis. ${ }^{1}$ It is a very rare disease. There are approximately 500 to 550 case reports in the literature..$^{2,3}$ Clinically, the patients may be either asymptomatic or symptomatic due to the involvement of multiple organs. Symptomatic ECD can be in different forms according to system involvement as cardiac, central nervous system, pulmonary, neuroendocrine, orbital-craniofacial, retroperitoneal, or multisystem dominant. ${ }^{2}$ ECD should be suspected by the radiological findings of skeletal system besides the clinical findings of multisystem involvement. Definite diagnosis can be made by CD68(+) CD1a(-) histiocytes seen in biopsy specimen. ${ }^{4}$ Treatment should be administered by experienced professionals in ECD. ${ }^{2}$

\section{CASE REPORT}

A 51-year-old male patient admitted to our outpatient clinic with complaints of flank pain and fatigue. He had a history of renal stones and peripheral arterial disease. He had a medically unmanageable hypertension. Blood pressure was 200/110 mmHg. Serum creatinine level was $1.2 \mathrm{mg} / \mathrm{dL}$. He was evaluated with contrastenhanced multislice computed tomography. Along with small calyceal stones, there was bilateral, quite symmetrical perinephric "rind-like" soft tissue infiltration. Right kidney was atrophic but still functioning without pelvicalyceal contrast excretion. The soft tissue infiltration obliterated both renal hila and caused proximal ureteral stenoses with mild calyceal dilation on the left side (Figures 1 and 2). Distally, the ureters were normal. Incidentally, abdominal aorta, common iliac arteries, proximal celiac trunk, and proximal renal arteries were noticed to appear "coated" with subtle perivascular tissue infiltration. An endovascular aortoiliac stent-graft was in place. $\mathrm{He}$ rejected any further diagnostic intervention after he presented with a blood creatinine of $2.2 \mathrm{mg} / \mathrm{dL}$. He underwent an unenhanced 


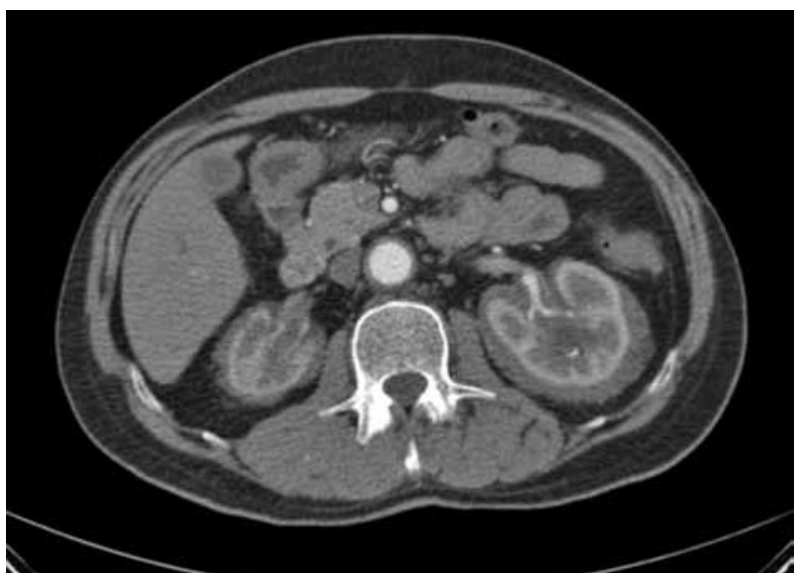

Figure 1. Arterial phase contrast-enhanced axial computed tomography scan shows rind-like soft tissue of perinephric space and also surrounding the aorta.

computed tomography examination with the same findings before. Renal scintigraphy revealed a non-functioning right kidney. Perinephric soft tissue infiltration was considered as the differential diagnosis of ECD. Direct radiographies of the extremities were obtained. All of the long tubular bones showed diametaphyseal cortical and patchy medullary sclerosis reinforcing the diagnosis of ECD (Figure 3). The patient underwent right nephrectomy for the control of hypertension and a double-J stent was placed on the left side for the relief of ureteral obstruction. The nephrectomy specimen showed the typical histological features of ECD. Serum creatinine level dropped to the borderline values after the operation and his blood pressure was in the normal range with medications. The pathological analysis of the nephrectomy specimen revealed an inflammatory infiltrate formed by foamy histiocytes that were positively stained for CD68 and neutrophilic aggregates which confirmed the preoperative diagnosis (Figures 4 and 5). A written informed consent was obtained from the patient.

\section{DISCUSSION}

The X-ray changes that are considered to be pathognomonic and found in almost all patients are dia-metaphyseal osteosclerosis bilaterally and symmetrically in the tubular bones of upper and lower extremities. ${ }^{5}$ They also show an increased uptake of tracer on Technetium $99 \mathrm{~m}$

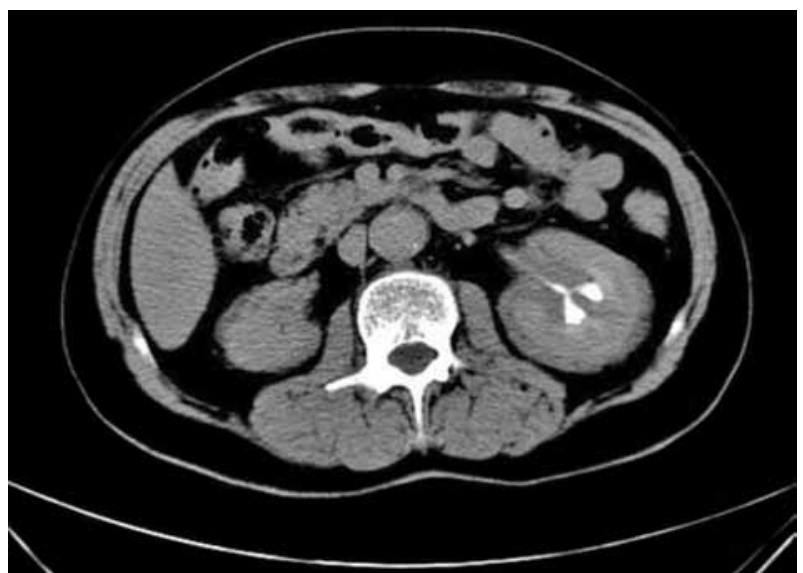

Figure 2. On delayed-phase computed tomography scan, the right kidney is not excreting intravenous contrast agent. On the left, the ureter and renal pelivis is encased with soft tissue infiltrate.

bone scintigraphy. ${ }^{4}$ These bone changes may be misdiagnosed as Paget's disease, lymphoma, sarcoidosis, metastases, or lipid storage diseases. ${ }^{6,7}$

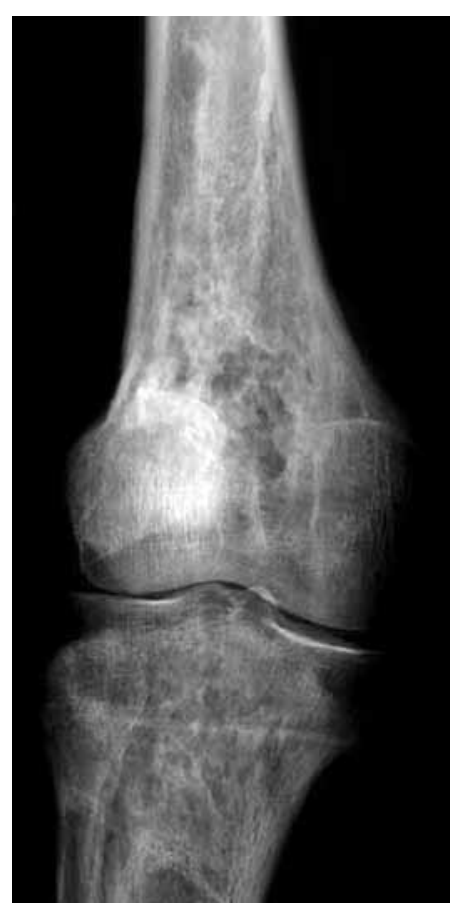

Figure 3. Anteroposterior radiogram of the right knee shows patchy sclerosis and coarse trabecular pattern of the femoral metadiaphysis. The same findings are present on both knees and upper extremities (not shown). 


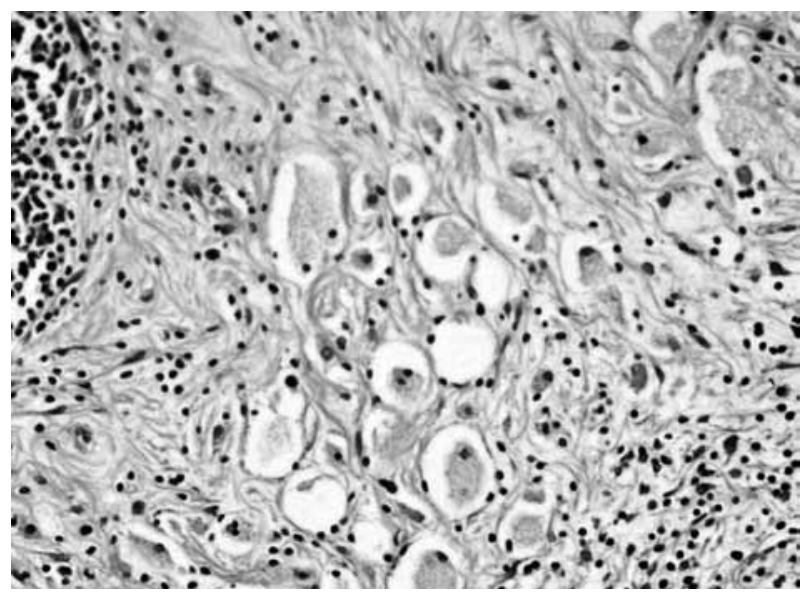

Figure 4. Light microscopy shows foamy histiocytes, neutrophil aggregates forming inflammatory infiltrate $(\mathrm{H}-\mathrm{E} \times 40)$.

The histiocytic infiltrative process, as found in our case, may involve the retroperitoneum in approximately 29 to $68 \%$ of patients. ${ }^{4,5}$ The soft tissue infiltrate around the aorta and its major branches can be misdiagnosed as arteritis, lymphoma or retroperitoneal fibrosis. ${ }^{4}$ The process may lead to severe arterial stenosis and the consequences include cerebral ischemia, cardiac angina, mesenteric ischemia, or renovascular hypertension. ${ }^{6-8}$ Adrenal glands, kidneys, renal arteries, ureters, and adjacent anatomical spaces may be involved in the process. Infiltration of perirenal space may extend to the pararenal space with irregular borders, thus will be given the name "hairy kidney". ${ }^{6}$ Extension to the renal sinuses and pedicles, proximal and distal ureters is possible and may cause obstruction and eventually, as in our case, compromise renal function. ${ }^{8-10}$

In our case, computed tomography findings suggested retroperitoneal fibrosis but the infiltrate did not involve the vena cava and the paraaortic infiltration was circumferential instead of sparing the posterior aspect of the aorta. Also, the perinephric infiltration was far more striking than the perivascular space which is inconsistent with retroperitoneal fibrosis. Therefore, the differential diagnosis consisted mainly of processes involving perinephric and perivascular spaces, namely, lymphoma, retroperitoneal fibrosis, and ECD. The diagnosis of ECD was strongly suggested with the radiographic findings of the extremities which revealed symmetrical dia-metaphyseal osteosclerosis.

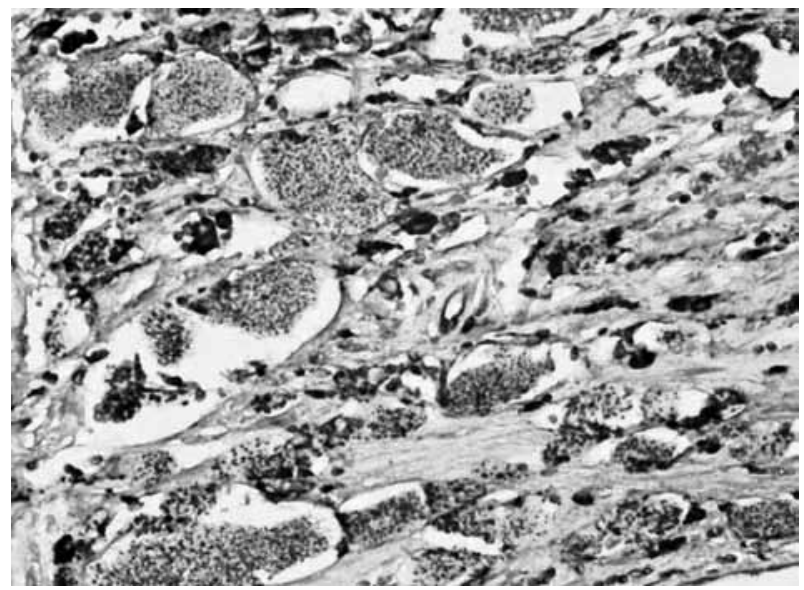

Figure 5. Immunocytochemistry shows foamy histiocytes with positive stain for CD68, which is pathognomonic for non-Langerhans cell histiocytes.

The final and definite diagnosis was confirmed with pathological evaluation of the nephrectomy specimen. The typical nonLangerhans' foamy histiocytes that lacked Birbeck granules, located in a polymorphic granuloma with xanthogranulomatosis was shown. Immunohistochemical staining revealed CD68(+), CD1a(-), and S-100 (-).

In conclusion, ECD should to be remembered in the differential diagnosis of retroperitoneal processes involving the major vascular structures, ureters, and perinephric space. Direct radiographic evaluation of the long tubular bones can be helpful to identify the systemic disease. The immunohistochemical studies of the proper tissue specimens may provide the definite diagnosis.

\section{Declaration of conflicting interests}

The authors declared no conflicts of interest with respect to the authorship and/or publication of this article.

\section{Funding}

The authors received no financial support for the research and/or authorship of this article.

\section{REFERENCES}

1. Veyssier-Belot C, Cacoub P, Caparros-Lefebvre D, Wechsler J, Brun B, Remy M, et al. Erdheim-Chester disease. Clinical and radiologic characteristics of 59 cases. Medicine (Baltimore) 1996;75:157-69. 
2. Diamond EL, Dagna L, Hyman DM, Cavalli G, Janku F, Estrada-Veras J, et al. Consensus guidelines for the diagnosis and clinical management of ErdheimChester disease. Blood 2014;124:483-92.

3. Ding H, Li Y, Ruan C, Gao Y Wang H, Zhang X, Liao Z. Chinese Erdheim-Chester disease: clinicalpathology-PET/CT updates. Endocrinol Diabetes Metab Case Rep 2015;2015:150055.

4. Mazor RD, Manevich-Mazor M, Shoenfeld Y. ErdheimChester Disease: a comprehensive review of the literature. Orphanet J Rare Dis 2013;8:137.

5. Dion E, Graef C, Miquel A, Haroche J, Wechsler B, Amoura Z, et al. Bone involvement in Erdheim-Chester disease: imaging findings including periostitis and partial epiphyseal involvement. Radiology 2006;238:632-9.
6. Dion E, Graef C, Haroche J, Renard-Penna R, CluzelP, Wechsler B, et al. Imaging of thoracoabdominal involvement in Erdheim-Chester disease. AJR Am J Roentgenol 2004;183:1253-60.

7. Castle EP, Humphreys MR, Andrews PE. Laparoscopic biopsy and ureterolysis in Erdheim-Chester disease. Mayo Clin Proc 2005;80:546-8.

8. Venkatanarasimha N, Garrido MC, Puckett M, White P. AJR teaching file: A rare multisystem disease with distinctive radiologic-pathologic findings. AJR Am J Roentgenol 2009;193:49-52.

9. Fortman BJ, Beall DP. Erdheim-Chester disease of the retroperitoneum: a rare cause of ureteral obstruction. AJR Am J Roentgenol 2001;176:1330-1.

10. Lee JH, Stodell M, Fowler JC. Renal bone disease. J R Soc Med 2005;98:165-6. 